\title{
FIRST STEPS IN DESCRIPTIVE THEORY OF LOCALES
}

\author{
JOHN ISBELL
}

\begin{abstract}
F. Hausdorff and D. Montgomery showed that a subspace of a completely metrizable space is developable if and only if it is $F_{\sigma}$ and $G_{\delta}$. This extends to arbitrary metrizable locales when " $F_{\sigma}$ " and " $G_{\delta}$ " are taken in the localic sense (countable join of closed, resp. meet of open, sublocales). In any locale, the developable sublocales are exactly the complemented elements of the lattice of sublocales. The main further results of this paper concern the strictly pointless relative theory, which exists because-always in metrizable localesthere exist nonzero pointless-absolute $G_{\delta}$ 's, $G_{\delta}$ in every pointless extension. For instance, the pointless part $\mathrm{pl}(\mathbf{R})$ of the real line is characterized as the only nonzero zero-dimensional separable metrizable pointless-absolute $G_{\delta}$. There is no nonzero pointless-absolute $F_{\sigma}$. The pointless part of any metrizable space is, if not zero, second category, i.e. not a countable join of nowhere dense sublocales.
\end{abstract}

\section{INTRODUCTION}

This paper initiates, not a generalization of descriptive set theory, but a reformulation of it admitting "pointless" subsets or sublocales of a metrizable space. An open, or closed, or $F_{\sigma}$ sublocale is a subspace-open, closed, or $F_{\sigma}$ respectively. However, the countable intersections of open sublocales, in the lattice of sublocales, are not in general subspaces. We call them $O_{\delta}$ 's. In completely metrizable spaces, $O_{\delta}$ is the same as $G_{\delta}$. In general, any $O_{\delta}$ which is a subspace is a $G_{\delta}$. One may think of the $O_{\delta}$ 's as (1) those which are subspaces: the nice $G_{\delta}$ 's, and (2) the others. The set of points of an $O_{\delta}$ is a $G_{\delta}$, but two different $O_{\delta}$ 's can have the same set of points.

This first paper does not go much beyond a study of $O_{\delta}$ 's. Hausdorff and Montgomery showed $[\mathrm{K}]$ that in a completely metrizable space, the subspaces which are both $F_{\sigma}$ and $G_{\delta}$ coincide with those which can be expanded in an alternating series of closed sets,

$$
\left.\left.\cdots\left(\left((\cdots)\left(\left(F_{0} \backslash F_{1}\right) \cup F_{2}\right) \backslash F_{3}\right) \cup \cdots\right) \cup F_{\omega}\right) \backslash F_{\omega+1}\right) \cup \cdots,
$$

with $\left\{F_{\alpha}\right\}$ decreasing (nonstrictly). It turns out that completeness is unnecessary, indeed spatiality is unnecessary for the Hausdorff-Montgomery theorem; it holds in metrizable locales with " $O_{\delta}$ " in place of " $G_{\delta}$ ".

Received by the editors August 18, 1989.

1980 Mathematics Subject Classification (1985 Revision). Primary 54H05; Secondary 06D20.

Partially supported by the Caribbean Mathematics Foundation. 
In arbitrary locales, the complemented elements of the lattice of sublocales are exactly the sublocales which can be expanded in an alternating series of closed sublocales. Thus

In metrizable locales, the complemented sublocales are the same as the sublocales which are at once $F_{\sigma}$ and $O_{\delta}$.

That is much the best result of the paper. The most interesting of the further results are (1) in metrizable locales, pointless absolute $O_{\delta}$ 's exist: a pointless metrizable locale is $O_{\delta}$ in every pointless metrizable extension if and only if it is the pointless part (= largest pointless sublocale) of some completely metrizable space. But $\left(1^{\prime}\right)$ this does not extend to dense embeddings in pointless completely regular locales, as "absolute $G_{\delta}$ " does for spaces. (2) There are no nonzero pointless absolute $F_{\sigma}$ 's. (3) The pointless part of the real line is characterized as the only nonzero zero-dimensional pointless metrizable absolute $O_{\delta}$ with a countable base. (4) The pointless part of any nonzero metrizable space (complete or not) is second category, i.e. not a countable join of nowhere dense sublocales.

\section{Complements}

In metrizable locales there seems to be a working descriptive theory; in more generality, fragments. But the fragments are numerous, so we consider general locales, as far as is easily possible. The needed background is almost all in $\left[\mathrm{J}_{1}\right]$, if you can stand the point of view: Johnstone's iocales keep intruding their frames into innocent conversation, rather as if people were continually showing you their skeletons-

\section{"Webster was much possessed by death And saw the skull beneath the skin."}

For me, a locale $A$ has a frame $T(A)$ of open parts (sublocales) and a lattice $S(A)$ of all sublocales. $S(A)$, upside down, is the frame $T\left(A_{d}\right)$ of the dissolution locale $A_{d}$, which has a distinguished monomorphism to $A$ representing the sublocales of $A$ by their pullbacks (intersections) in $A_{d}$, which are precisely the closed sublocales of $A_{d}\left[\mathrm{I}_{1}, \mathrm{~J}_{2}\right] . S(A)^{\text {op }}$ is not just a frame but a zero-dimensional frame; more fully, if $x \neq y$ in $S(A)$, there is a locally closed sublocale $U \Delta V$ of $A$ (symmetric difference of two open parts) which has zero meet with one of $x$ and $y$ but not with the other. (This holds just because $x$ and $y$ are determined by the respective relations $U \wedge x=V \wedge x$, $U \wedge y=V \wedge y$, on $T(A)$.) Zero-dimensionality follows, for locally closed sublocales have complements.

There are three properties equivalent to complementedness in any locale. A developable sublocale is a sublocale

$$
F_{0}-F_{1} \vee F_{2}-F_{3} \vee \cdots \vee F_{\omega}-F_{\omega+1} \vee \ldots,
$$

where $\left\{F_{\alpha}\right\}$ is a transfinite descending sequence of closed sublocales. This notation (standard for spaces $[\mathrm{K}]$ ) is interpreted in the obvious way from the 
left, $\left(\left(\left(F_{0}-F_{1}\right) \vee F_{2}\right)-F_{3}\right) \vee \ldots$, and at a limit ordinal, intersect the descending initial segments $F_{0},\left(F_{0}-F_{1}\right) \vee F_{2}, \ldots$. ("-" means "meet with the complement of".) For a development $(*)$ one also requires continuity at limit ordinals $\lambda$ : $F_{\lambda}=\bigwedge_{\kappa<\lambda} F_{\kappa}$. (If it were omitted, it could be restored by increasing the length by 2, interpolating ' $\vee M-M$ ' at each limit position, where $M$ is the required term.) Second, we need the operation ', where $S^{\prime}$ is the meet of $S$ with the closure of the join of all sublocales of $S^{-}$disjoint from $S$ (telegraphically, $\left.S^{\prime}=S \wedge\left(S^{-} \mid S\right)^{-}\right)$. Put $S_{0}=S, S_{\alpha+1}=S_{\alpha}^{\prime}, S_{\lambda}=\Lambda_{\kappa<\lambda} S_{\kappa}$ at limit ordinals $\lambda$. Third, say that $S$ splits $T$ inseparably if $(S \wedge T)^{-} \wedge(T \backslash S)^{-} \supset T$. (Precisely, no closed proper sublocale of $T$ contains $S \wedge T$ or contains all sublocales of $T$ disjoint from $S$.)

1.1. Theorem. For a sublocale $S$ of a locale $A$, the following are equivalent:

(1) $S$ is a complemented sublocale.

(2) $S$ splits no nonzero (closed) sublocale inseparably.

(3) $S$ is a developable sublocale.

(4) $S_{\alpha}=0$ for some ordinal $\alpha$.

The proof depends on a lemma about developments, 1.2. For classical developments in subspaces, the operations $U$ and $\backslash$ are performed in a lattice of all subsets and the lemma is evident. Here, recall that limit ordinals are even. ( $2 \alpha$ is not $\alpha 2$, but is the order type of $\alpha$ blocks of 2.) Recall also that every locale $A$ has a smallest dense sublocale $D(A),\left[\mathrm{I}_{1}\right.$ or $\left.\mathrm{J}_{1}\right]$.

1.2. Lemma. For a development in complemented sublocales,

$$
F_{0}-F_{1} \vee F_{2}-F_{3} \vee \cdots \vee F_{\omega}-F_{\omega+1} \vee \ldots,
$$

indexed by the ordinals less than a limit ordinal $\lambda$, let $M$ be the meet of the even initial segments, $F_{0}, F_{0}-F_{1} \vee F_{2}, \ldots$; let $J$ be the join of the odd initial segments $F_{0}-F_{1}, F_{0}-F_{1} \vee F_{2}-F_{3}, \ldots$ Then $J \vee\left(\bigwedge_{\kappa<\lambda} F_{\kappa}\right)=M$.

Proof. Just as for alternating series of real numbers, each odd initial segment is $\leq$ each even initial segment, so $J \leq M$; and evidently $\left(\bigwedge_{\kappa<\lambda} F_{\kappa}\right) \leq M$. So we need only show that if $z \wedge M \neq 0$ then $z$ meets $J$ or $\bigwedge_{\kappa<\lambda} F_{\kappa}$. But if $z \wedge M$ does not meet $J$, it never meets $F_{0}-F_{1} \vee \cdots \vee F_{2 \alpha}-F_{2 \alpha+1}$. It is, however, $\leq M \leq\left(F_{0}-F_{1} \vee \cdots \vee F_{2 \alpha}-F_{2 \alpha+1}\right) \vee F_{2 \alpha+2}$. Hence $z \wedge M \leq F_{2 \alpha+2} \leq F_{2 \alpha+1}$; $z \wedge M \leq F_{\kappa}$ for all $\kappa<\lambda$, so $z \wedge M \leq \bigwedge_{\kappa<\lambda} F_{\kappa}$.

Proof of 1.1. If (1) $S$ has a complementary sublocale $C$, and $T$ is a nonzero sublocale, $D(T)$ is nonzero. Not both $S$ and $C$ contain $D(T)$; hence $S \wedge T$ or $C \wedge T$ is not dense in $T$, so $S$ does not split it inseparably.

Assume (2). Then every nonzero closed sublocale $T$ has a closed proper part $T^{*}$ which contains either $S \wedge T$ or $(T \backslash S)^{-}$[all parts of $T$ disjoint from $S]$. Put $E_{0}=A, E_{\alpha+1}=E_{\alpha}^{*}, E_{\lambda}=\bigwedge_{\kappa<\lambda} E_{\kappa}$ at limit ordinals. Evidently the descending sequence $\left\{E_{\alpha}\right\}$ must reach 0 , at some limit ordinal.

We now construct a subsequence of $\left\{E_{\alpha}\right\}$ which will be a development of $S$ (discontinuous. As noted above, a continuous development can be obtained by 
interpolating suitable pairs of terms $\vee M-M) . E_{0}=A$ contains $S$. Let $F_{0}$ be the last $E_{\alpha}$ which contains $S$; since $\left\{E_{\alpha}\right\}$ is continuous at limit ordinals and has a last term, $F_{0}$ exists. If $F_{0}=0$, stop. Otherwise $F_{0}$ is some $E_{\beta}$, and $E_{\beta+1}=F_{0}^{*}$ does not contain $S \wedge F_{0}=S$, so it does contain $\left(F_{0} \backslash S\right)^{-}$. Let $F_{1}$ be the last $E_{\alpha}$ which contains $\left(F_{0} \backslash S\right)^{-}$. [At this point the reader may prefer to amplify "and so on" in his own notation.] Inductively, stopping whenever we are out of $E$ 's $\left(F_{\alpha}=0\right)$, with each $F_{2 \alpha+2}$ being the last $E_{\beta}$ which contains $S \wedge F_{2 \alpha+1}$ and $F_{2 \alpha+1}$ the last $E_{\beta}$ which contains $\left(F_{2 \alpha} \backslash S\right)^{-}$, the successor $E_{\beta+1}$ contains the other (i.e., respectively $\left(F_{2 \alpha+2} \backslash S\right)^{-}$or $\left.S \wedge F_{2 \alpha+1}\right)$. Let (respectively) $F_{2 \alpha+3}$ be the last $E_{\gamma}$ containing $\left(F_{2 \alpha+2} \backslash S\right)^{-}$, and $F_{2 \alpha+2}$ the last $E_{\gamma}$ containing $S \wedge F_{2 \alpha+1}$. Having-as we do for $F_{0}$ and $F_{0}-F_{1}$-every even initial segment $I_{2 \delta}$ of the development containing $S$ and every odd one $I_{2 \delta+1}$ contained in $S$, these extensions preserve those inclusions, as follows. $I_{2 \alpha+3}=I_{2 \alpha+1} \vee F_{2 \alpha+2}-F_{2 \alpha+3}$, removing $F_{2 \alpha+3}$ and thus $\left(F_{2 \alpha+2} \backslash S\right)^{-}$, so still $I_{2 \alpha+3} \leq S$. And $I_{2 \alpha+2}=I_{2 \alpha}-F_{2 \alpha+1} \vee F_{2 \alpha+2}$, putting back $F_{2 \alpha+2} \geq S \wedge F_{2 \alpha+1}$, so still $I_{2 \alpha+2} \geq S$.

At a limit ordinal $\lambda$, observe that vacuously (as for $\lambda=0$ ) the next $E$, which is $\bigwedge_{\kappa<\lambda} F_{\kappa}$, contains $S \wedge\left(\bigwedge_{\kappa<\lambda} F_{\kappa}\right)$. Let $F_{\lambda}$ be the last $E_{\gamma}$ which does so, and let $F_{\lambda+1}$ be the last $E_{\gamma}$ which contains $\left(F_{\lambda} \backslash S\right)^{-}$. As before, $I_{\lambda} \geq S$ and $I_{\lambda+1} \leq S$. The induction runs. It can only terminate with a last term 0 or with no last term but 0 intersection. In either case (in view of 1.2) we have a development of $S$.

For $(3) \Rightarrow(4)$, if $S$ has a (continuous) development $F_{0}-F_{1} \vee F_{2}-F_{3} \vee \cdots$ of a certain length $\alpha$ (i.e. $F_{\alpha}=0$ or the indices are the predecessors of $\alpha$ ), we shall see that $S_{\alpha}=0$. First, $S_{1}=S \wedge\left(S^{-} \backslash S\right)^{-}$has a development $G_{0}-$ $G_{1} \vee G_{2}-G_{3} \vee \cdots$ where $G_{\alpha}=F_{\alpha} \wedge\left(S^{-} \backslash S\right)^{-}$. But further, the first two terms can be omitted, because $\left(F_{0}-F_{1}\right) \wedge\left(S^{-} \backslash S\right)^{-}=0$; that follows from $S^{-} \leq F_{0}$, $F_{0}-F_{1} \leq S$, and thence $\left(S^{-} \backslash S\right)^{-} \leq F_{1}$. Now by an evident induction each $S_{\beta}$ has a development $H_{2 \beta}-H_{2 \beta+1} \vee \cdots$, where $H_{\gamma}=F_{\gamma} \wedge K_{\beta}, K_{\beta}$ being $\left(S_{\beta-1}^{-} \mid S_{\beta-1}\right)^{-}$if $\beta$ is a successor or $\bigwedge_{\gamma<\beta} K_{\gamma}$ if $\beta$ is a limit ordinal. So $S_{\alpha}=0$.

Assume $S_{\alpha}=0$. Then for $S$ to be complemented it will suffice that each $S-S_{\beta}$ is complemented. ( $S_{\beta}$ is complemented in $S$, being relatively closed.) Now $S-S_{1}=S-\left(S^{-} \backslash S\right)^{-}=S^{-}-\left(S^{-} \backslash S\right)^{-}$, open in a closed sublocale of $A$, thus complemented. Putting $S_{\beta}$ for $S, S_{\beta}-S_{\beta+1}$ is complemented; so $S-S_{\beta+1}$ is complemented if $S-S_{\beta}$ is. For a limit ordinal $\lambda$, the complements $C_{\kappa}$ of $S-S_{\kappa}(\kappa<\lambda)$ are determined by $C_{\kappa} \wedge S=S_{\kappa}, C_{\kappa} \vee S=1$ in $S(A)$. Put $C_{\lambda}=\wedge_{\kappa<\lambda} C_{\kappa}$. We have $C_{\lambda} \vee S=1$ since $S(A)$ is anti-isomorphic with a frame. And $C_{\lambda} \wedge S=\left(\wedge C_{\kappa}\right) \wedge S=\wedge\left(C_{\kappa} \wedge S\right)=\wedge S_{\kappa}=S_{\lambda}$, so $C_{\lambda}$ is the complement of $S-S_{\lambda}$. The induction runs; the proof is finished.

The equivalence of (2), (3), and (4) for subspaces of a space is classical [K]. 


\subsection{A complemented sublocale of a space is a subspace.}

Proof. If $B$ and $C$ are complements in $S(X)$, every point is in $B$ or in $C$; the join $B_{-}$of the points in $B$ satisfies $B_{-} \vee C=X$ since $B_{-} \vee C$ contains all the points; since $B_{-} \wedge C=0, B_{-}=B$, a subspace.

1.4. Every complemented sublocale of a regular locale is a join of closed sublocales; in fact, in any locale $A$, when a meet $m$ of complemented sublocales $x_{\alpha}$ is complemented, $1-m=\bigvee\left(1-x_{\alpha}\right)$.

Proof. The second clause implies the first, since every sublocale of a regular locale is a meet of open sublocales $\left[\mathrm{I}_{1}\right]$. To prove it, let $c=\mathrm{V}\left(1-x_{\alpha}\right)$. Each $c \vee x_{\alpha}=\bigvee\left(1-x_{\beta}\right) \vee x_{\alpha} \geq 1-x_{\alpha} \vee x_{\alpha}=1$. Then, since $S(A)$ is anti-isomorphic with a frame, the join $c \vee m=c \vee\left(\bigwedge x_{\alpha}\right)=\Lambda\left(c \vee x_{\alpha}\right)=\Lambda 1=1$. Given the existence of $1-m$, from $c \vee m=1$ we have $c \geq 1-m$ and from $m \leq x_{\alpha}$ we have $1-m \geq 1-x_{\alpha}$, so $1-m \geq \bigvee\left(1-x_{\alpha}\right)=c$. Thus $1-m=c$.

Of course 1.4 fails for frames; in fact, it fails in frames anti-isomorphic with sublocale lattices $S(A)$. That is, in $S(A)$ itself, a join $j$ of complemented elements $x_{\alpha}$ can have a complement which is not $\Lambda\left(1-x_{\alpha}\right)$. Let $A$ be a dense-in-itself Hausdorff space and the $x_{\alpha}$ the singletons; then $\bigvee x_{\alpha}=1$ has complement 0 , but $\Lambda\left(1-x_{\alpha}\right) \supset D(A) \neq 0$.

The main application of the formula in 1.4 is that the complement of a " $G_{\delta}$ ", if it exists, is an $F_{\sigma}$. Which brings up $F_{\sigma}$ and all that. We call a countable meet, in $S(A)$, of open sublocales an $O_{\delta}$ sublocale-because when $A$ is a space, " $G_{\delta}$ " has an established meaning. However, a countable join of closed sublocales is simply an $F_{\sigma}$ sublocale. In a space, closed sublocales are subspaces $(=$ joins of their points), so an $F_{\sigma}$ sublocale is also a subspace; moreover, clearly, the $F_{\sigma}$ sublocales are exactly the $F_{\sigma}$ subspaces. As for $G_{\delta}$ and $O_{\delta}$, the relationship is controlled by

1.5. A sublocale $B$ of a regular locale $A$ is the meet of sublocales $S_{i}$ containing it if and only if for every nonzero closed part $J$ disjoint from $B$, not all $S_{i} \wedge J$ are dense in $J$.

Proof. Clearly if all $S_{i} \wedge J$ are dense, they and $\wedge S_{i}$ contain $D(J)$, which is nonzero and contained in complemented $J$, disjoint from $B$. Conversely, suppose $C=\bigwedge S_{i} \neq B$. Since $C>B$, there is a nonzero symmetric difference $L$ of open parts of $A$ which meets $C$ but not $B$. Now $L$ is locally closed; thus there are closed parts $L_{\alpha}$ of $A$ contained in $L$ whose relative interiors $I_{\alpha}$ cover $L . \quad L \wedge C=\bigvee\left(I_{\alpha} \wedge C\right.$ ) (by 1.0 of $\left[I_{3}\right]$ : a sublocale is the join of a relatively open cover), so some $I_{\alpha} \wedge C \neq 0 \neq L_{\alpha} \wedge C$. Consider $J=\left(L_{\alpha} \wedge C\right)^{-}$: nonzero closed, contained in $L_{\alpha}$ and thus disjoint from $B$, with $J \wedge C$ dense in $J$. Then every $J \wedge S_{i}$ is dense in $J$.

The further Borel types will be called $F_{\sigma \delta}, O_{\delta \sigma}$, and so on. An $F_{\sigma \delta}$ is a countable sublocale meet of $F_{\sigma}$ 's, so not the same in spaces as a classical $F_{\sigma \delta}$; so one must say "classical $F_{\sigma \delta}$ " or " $F_{\sigma \delta}$ sublocale". 
It is known $\left[\mathrm{I}_{2}\right]$ that in locally compact spaces, every $G_{\delta}$ is an $O_{\delta}$; more, every $O_{\delta}$ is a subspace. (So a $G_{\delta}$, being the set of points of an $O_{\delta}$, which is a subspace, is that subspace. Seemingly the converse could fail; every $G_{\delta}$ might be $O_{\delta}$, but some nonspatial sublocales could also be $O_{\delta}$.) Therefore this is true in $G_{\delta}$ subspaces of locally compact spaces. For $T_{3}$ spaces, the following is a little more general:

1.6. In a regular space in which every nonempty closed subspace is second category in itself, every $O_{\delta}$ sublocale is a subspace (so every $G_{\delta}$ is an $O_{\delta}$ ).

Proof. Let $C=\wedge U_{i}$ be an $O_{\delta}$ sublocale $\left(U_{i}\right.$ open) and consider the subspace $B$ on $\cap U_{i}$. If $J$ is a nonzero closed set disjoint from $B$, not all $U_{i} \cap J$ are dense in $J$ since then they would have a common point. Hence $B=\wedge U_{i}=C$.

1.7. A closed $G_{\delta}$ set in a normal space is an $O_{\delta}$ subspace.

Proof. If closed $H$ is $\cap U_{i}$ ( $U_{i}$ open) it is $\bigcap V_{i}$ where $H \subset V_{i} \subset V_{i}^{-} \subset U_{i}$. Hence $H \leq \wedge V_{i} \leq \wedge V_{i}^{-}=H$, so $H$ is the $O_{\delta}$ sublocale $\wedge V_{i}$.

1.8. There is a closed $G_{\delta}$ set in a completely regular space which is not an $O_{\delta}$. Proof. In the compact Hausdorff space $[0,1] \times\left(\omega_{1}+1\right)$ (where $\omega_{1}+1$ has the order topology) consider $X=\left(P \times\left\{\omega_{1}\right\}\right) \cup\left(Q \times \omega_{1}\right)$, where $Q$ is a countable dense set in $[0,1]$ and $P=[0,1] \backslash Q . P \times\left\{\omega_{1}\right\}$ is closed in $X$, and $Q \times \omega_{1}$ is $F_{\sigma}$ since $\{q\} \times \omega_{1}$ is closed for each $q \in Q$. So $P \times\left\{\omega_{1}\right\}$ is a $G_{\delta}$ set. But a neighborhood of $\left(p, \omega_{1}\right)$ contains $U \times T$ for a $[0,1]$-neighborhood $U$ of $p$ and a tail $T$ of $\omega_{1}$. A collection of these covering $P \times\left\{\omega_{1}\right\}$ has a countable subcover, so it contains $V \times T$ for some neighborhood $V$ of $P$ and some tail $T$ of $\omega_{1} . V \times T$ is dense in $[0,1] \times T$; and from 1.5 , clearly $P \times\left\{\omega_{1}\right\}$ is not an $O_{\delta}$ sublocale.

Observe, by 1.4, the complement of an $O_{\delta}$ (which has a complement) is an $F_{\sigma}$. By 1.8, the complement of an $F_{\sigma}$ need not be $O_{\delta}$. There is no such example in metrizable locales, because Hausdorff and Montgomery showed, in the complete case, that developable (= complemented) subspaces must be $F_{\sigma}$ and $G_{\delta}[K]$, and this extends to the general case by means of two pullback lemmas, 1.9, 1.10.

1.9. For a locale morphism $f: A \rightarrow B$ and a sublocale $L \subset B$, if $L$ is open, or closed, or locally closed, or $O_{\delta}$, then so is the pullback $f^{-1}(L) \subset A$. If $f$ is an embedding then every open/closed/locally closed $/ O_{\delta}$ sublocale of $A$ is of this form.

Proof. It is simple routine to verify that the pullback $f^{-1}(L)$ for $L$ open is the open sublocale $f^{*}(L)$. Hence for $L$ closed, $f^{-1}(L)$ is closed $A-f^{*}(B-L)$; and similarly for locally closed parts $(=$ open $\wedge$ closed $)$. These verifications are largely done in the proof of functoriality of the sublocale lattice $S\left(\right.$ ) $\left[\mathrm{I}_{1}\right.$, 1.4]; and it is functoriality to upside-down frames, i.e. $f^{-1}$ preserves infinite meets-hence $O_{\delta}$ 's. As for embeddings, they are defined by surjectiveness of 
$f^{*}$, i.e. every open part of $A \subset B$ is of the indicated form. Since $f^{-1}$ preserves meets and complements, the rest follows.

From 1.9, a zero set of a real-valued continuous function is an $O_{\delta}$, which generalizes 1.7. A closed $O_{\delta}$ in a completely regular space need not be a zero set, and there is a painfully cute example. The familiar Tychonoff plank has a long side $L$ and a short side $S$, disjoint but without disjoint neighborhoods; but since $S$ is short, $L$ is a zero set. So wedge two planks, $S$ to $S$. Each copy of $L$ is still a closed $O_{\delta}$ but no longer a zero set.

1.10. If $A$ is a sublocale of $B$, every complemented sublocale of $A$ is $C \wedge A$ for some complemented sublocale $C$ of $B$.

Proof. A complemented sublocale $D$ of $A$ is developable,

$$
D=F_{0}-F_{1} \vee F_{2}-F_{3} \vee \cdots
$$

for some descending transfinite sequence $\left\{F_{\alpha}\right\}$ of closed sublocales. Let $G_{\alpha}=$ $F_{\alpha}^{-}$in $B$. The development given by $\left\{G_{\alpha}\right\}$ expresses a complemented sublocale $C$ of $B$. The initial segments $I_{0}, J_{0}$ of the two developments $\left(I_{0}=F_{0}\right.$, $J_{0}=G_{0}$ ) satisfy $J_{0} \wedge A=I_{0}$; when this is true for $I_{\alpha}$ and $J_{\alpha}$ it is so for $\alpha+1$ too, and similarly at limit ordinals. Hence $C \wedge A=D$.

Now a metrizable locale $A$ can be embedded in a complete metric space $B$, where developable subspaces $C, B-C$ are $F_{\sigma}$ and $G_{\delta}$ (Hausdorff, Montgomery $[\mathrm{K}, \mathrm{M}])$. So they are $O_{\delta} ; C \wedge A, A-C$ are $O_{\delta}$, and by 1.4 they are $F_{\sigma}$.

\subsection{Complemented sublocales of a metrizable locale are $F_{\sigma}$ and $O_{\delta}$.}

The converse of 1.11 is also true. Of course it is known in complete spaces; this seems $[\mathrm{K}]$ to be due to Hausdorff alone. (For 1.11, the nonseparable case essentially depends on paracompactness, and Montgomery's proof [M] before paracompactness was thought of is fairly formidable. It is given also in $[\mathrm{K}]$.) The key to finishing this is

1.12. A nonzero metrizable locale cannot be split inseparably by a sublocale which is $F_{\sigma}$ and $O_{\delta}$.

Proof. First let $X$ be a metric space and $F, G=X \backslash F$ two dense $F_{\sigma}$ 's: $F=$ $\bigcup F_{i}, G=\bigcup G_{j}, F_{i}$ and $G_{j}$ closed. Since $F$ and $G$ are dense, each $G_{j}$ and each $F_{i}$ are nowhere dense.

We may assume $F_{i+1} \supset F_{i}, G_{j+1} \supset G_{j}$. We construct a nonzero closed subspace $C$ of $X$ contained in $F$, with each $C \backslash F_{i}$ dense in $C$. If this succeeds, it will show that $G$ is not $O_{\delta}$; for any sequence of open neighborhoods $U_{i}$ of $G$ with intersection $G$ gives us a representation of $F$ as $\bigcup\left(X \backslash U_{i}\right)$, and we shall always have a violation of 1.5.

Let $p_{1}$ be a point of $F$. Then $p_{1}$ is at positive distance $2 \varepsilon_{1}$ from $G_{1}$; all later choices of points $p_{j}, p_{j k}$ will be outside the $\varepsilon_{1}$-neighborhood of $G_{1}$. Let $p_{2}$ be a point of $F \backslash F_{2}$ within distance $2^{-1}$ of $p_{1}$. It is at positive distance $2 \varepsilon_{2}$ 
from $G_{2}$, and all later $p$ 's will be outside the $\varepsilon_{2}$-neighborhood of $G_{2}$. Having a finite set of points $p_{j k}$ of $F \backslash F_{j}$, they are at positive minimum distance $2 \varepsilon_{j}$ from $G_{j}$, and we shall stay (hereafter) outside the $\varepsilon_{j}$-neighborhood of $G_{j}$. In $F \backslash F_{j+1}$ (which is dense), choose a finite set of points $p_{j+1, k}$ including one within distance $2^{-j}$ of each preceding $p_{i m}$. Completing the induction, we have a countable subset $C_{0}=\left\{p_{j k}\right\}$ of $F$. Any convergent sequence in $C_{0}$ that is not finally constant is finally out of each finite subset of $C_{0}$, and therefore the limit is not in any $G_{j}$; so $C=C_{0}^{-} \subset F$. By construction, each $C_{0} \backslash F_{i}$ is dense in $C$.

Now observe that one can do nearly the same thing in a metric locale $A$, replacing points $p_{j k}$ with smallish closed sublocales $\pi_{j k}$. For instance, first choose points $p_{j k}$ of the completion outside $F_{j}$ and the proscribed neighborhoods of $G_{i}$ 's; then let $N_{j k}$ be the trace on $F$ of a suitably small closed neighborhood of $p_{j k}$, closed in $F$ and nonzero; and let $\pi_{j k}$ be the first nonzero $F_{i} \wedge N_{j k}$. (Since $N_{j k}$ is closed, $0 \neq N_{j k} \wedge\left(\bigvee F_{i}\right)=\bigvee\left(N_{j k} \wedge F_{i}\right)$.) Finitely many indices $k$ for each $j$ will no longer suffice, but $\left\{p_{j k}\right\}_{k}$ can be a set coming within $2^{-j}$ of all of $\bigvee_{i<j} \pi_{i m}$, with no two $p_{j k}$ twice that close to each other. Joins of discrete families of closed sublocales are closed. Again we find that $G$ is not $O_{\delta}$ in $A$.

1.13. Theorem. A sublocale of a metrizable locale is complemented if and only if it is $F_{\sigma}$ and $O_{\delta}$.

Proof. Necessity is in 1.11. If $B \subset A$ is $F_{\sigma}$ and $O_{\delta}$, so is $B \wedge C$ for each closed sublocale $C$, so by $1.12 B$ splits no such $C$ inseparably, and $B$ is complemented.

\section{Pointless PARTS}

It seems highly unlikely that locales without points are less diverse than topological spaces; but our descriptive vocabulary for them is much poorer. The pointless locales which are best known are the 'discrete' or Boolean ones, whose frame is a complete Boolean algebra. (Thus they can be defined by "Every open part is closed". In fact every part, i.e. sublocale, is open [and closed] [ $\left.\mathrm{I}_{1}\right]$.) They occur in every locale $A$, as its (smallest) dense part $D(A)$. As the heading indicates, we are going to look at pointless parts, meaning largest pointless parts of spaces. But first we look at the simple and definitive results on dense parts.

2.1. The Boolean sublocales of a space $X$ (or of a locale $A$ ) are precisely the sublocales $D(H)$ where $H$ is a closed subspace of $X$ (respectively a closed sublocale of $A$ ). If $H \neq H^{\prime}$ then $D(H) \neq D\left(H^{\prime}\right)$.

Proof. Every sublocale $B$ is dense in its closure $H$. If $B$ is Boolean, $D(H) \subset$ $B$, and $D(H)$ is dense in $B$, so $D(H)=B$. The correspondence is bijective since $H=B^{-}, B=D(H)$. 
2.2. The real line $\mathbf{R}$ has $\omega_{0}$ nonisomorphic Boolean sublocales, namely the discrete spaces of cardinal $n$ for $0 \leq n \leq \omega_{0}$, and $D(\mathbf{R})$ plus a discrete space of cardinal $n$ for those $n$.

Proof. Evidently $\mathbf{R}$ can be replaced by $[0,1]$. Each of its closed subspaces consists of the closure of the countable set $E$ of isolated points and an open set $U$ which is empty or locally compact dense-in-itself. If $U^{-} \neq 0$ it is an irreducible quotient of the Cantor set $\mathbf{C}$, and $D(U) \approx D(\mathbf{C}) \approx D(\mathbf{R})\left[\mathrm{I}_{4}\right]$; and $D(E)=E$, countable and discrete.

2.3. $D(\mathbf{R})$ is characterized, up to isomorphism, as a nonzero Boolean pointless separable metrizable locale.

Proof. If $A$ is such a locale, a metric completion of $A$ is separable, dense in itself, and without isolated points. Being separable, it has a metric compactification $F . F$ is an irreducible quotient of $\mathbf{C}$, and $A=D(F) \approx D(\mathbf{C}) \approx D(\mathbf{R})$.

The first thing about the largest pointless part is that it need not exist: the join of all pointless parts of a space may have points. Describing this (in order to avoid it) involves the weakly scattered spaces of Niefield and Rosenthal [NR], one definition for which is "Every nonzero sublocale has a point". There are various characterizations [NR].

2.4. The join $\mathrm{pl}^{+}(X)$ of the pointless sublocales of a sober space $X$ is the meet of the sublocales (subspaces) which have weakly scattered complements. Two open sets $U, V$ have the same trace on $\mathrm{pl}^{+}(X)$ if and only if the symmetric difference $U \Delta V$ is weakly scattered.

Proof. If $U \Delta V$ (which is open in its closure, and thus is a complemented sublocale) is weakly scattered, all pointless sublocales of $X$ and therefore their join are contained in the complement, so $U$ and $V$ have the same trace on $\mathrm{pl}^{+}(X)$. If $U \Delta V$ is not weakly scattered, not both of its parts $U-V, V-U$ (in the distributive lattice of sublocales) lack pointless parts; so $U$ and $V$ have different traces on some pointless part and therefore on $\mathrm{pl}^{+}(X)$. This shows that the join $\mathrm{pl}^{+}(X)$ is the meet of the complements of certain weakly scattered sublocales. But any sublocale with weakly scattered complement must contain $\mathrm{pl}^{+}(X)$, so it is the meet of all of them.

2.5. A sober space $X$ has a largest pointless sublocale $\mathrm{pl}(X)$ if and only if each point $x$ has a weakly scattered relative neighborhood in $\{x\}^{-}$.

Proof. The question is when no point $x$ is in $\mathrm{pl}^{+}(X)$. Now if $x$ has a weakly scattered relative neighborhood $N$ in $\{x\}^{-}, x$ is in the relative interior of $N$ which is disjoint from $\mathrm{pl}^{+}(X)$. If all relative neighborhoods of $x$ in $\{x\}^{-}$fail to be weakly scattered, they all have nonzero pointless sublocales. The meet of $\{x\}^{-}$and $\mathrm{pl}^{+}(X)$ is dense in the irreducible space $\{x\}^{-}$, so it contains $\{x\}^{-}$ and $x$.

In particular, if each point is open in its closure-e.g., closed- $\mathrm{pl}(X)$ exists. 
Remark. "Weakly scattered" is "without pointless part": pointlessnessless. So the meet of complements of weakly scattered subspaces is pointlessnesslessnessless. 2.5 tells us when it is simply pointless. Clearly in any case pointlessnesslessnesslessnessless $=$ pointlessnessless, or weakly scattered.

In particular, for $X \subset \mathbf{R}$ (in fact for $X$ sober $T_{1}$ ) we have $\mathrm{pl}(X)$. I have no idea how to characterize the locales which are $\mathrm{pl}$ (something) (like the Boolean locales which are $D$ (something)). It is not true (as it is for $D())$ that a sublocale of $X$ which is isomorphic with some $\operatorname{pl}(Y)$ is $\mathrm{pl}(Z)$ for some $Z \subset X$, even for complete separable metric $X$ (2.17.b below). We can, however, imitate 2.1 so far as to describe the pairs of subspaces $Y, Z$, for which $\operatorname{pl}(Y)=\operatorname{pl}(Z) \subset X$.

One would hope to characterize locales $A$ of the form $\mathrm{pl}(X)$ by constructing a suitable $X$ from $A$. (For $D()$, this is easily done.) In any case, what can we say about $X$, given $\operatorname{pl}(X)$ ? One such result is

\subsection{For $X$ regular and dense in itself, $\mathrm{pl}(X)$ has the same weight as $X$.}

Proof. Of course any sublocale $S$ has no greater weight, since the trace of a basis on $S$ is a basis for $S$. Conversely, if $X$ is dense in itself and $T_{1}, \operatorname{pl}(X)$ contains the smallest dense part; but in a regular space, that has the same weight.

Probably there is no closer relation between the number of points of $X$ and the size of $\operatorname{pl}(X)$, in any considerable generality, than the inequalities implied by 2.6. (For weight $w$ and power $p, w \leq 2^{p}$ and $p \leq 2^{w}$.) For $X$ metrizable and dense in itself, I do not know if $\operatorname{pl}(X)$ determines whether $X$ is countable, i.e. homeomorphic with $Q$. (Using 2.8, it is simple Polish routine to prove that $X$ is countable if an isomorphism of $\operatorname{pl}(X)$ and $\operatorname{pl}(Q)$ is induced by embeddings $X \subset Y, Q \subset Y$.) What I know about $\mathrm{pl}(Q)$ is in 2.17 and 2.18 .

A metrizable space is called totally imperfect if every Čech-complete subspace is scattered. Equivalently, it contains no Cantor set-for it is easy to construct a Cantor set in a complete dense-in-itself metric space. (In fact, this is done in 2.15 below, a bit more generally.) Countable complete metric spaces are scattered; scattered separable metric spaces are countable; so separable metrizable $X$ is totally imperfect iff all its Čech-complete subspaces are countable.

2.7. For a subspace $A$ of a space $X$, if $\operatorname{pl}(X \backslash A) \subset X$ is $\operatorname{pl}(X)$ then every subspace of $A$ closed in $X$ is weakly scattered, and the converse is true in regular spaces. Thus in completely metrizable $X, \operatorname{pl}(X \backslash A)=\operatorname{pl}(X)$ iff $A$ is totally imperfect.

Proof. From 2.4, $\operatorname{pl}(X \backslash A)=\operatorname{pl}(X)$ iff every locally closed $D \subset X$ with $D \backslash A$ weakly scattered is weakly scattered, which certainly implies that subsets of $A$ closed in $X$ are weakly scattered. If weakly scattered subsets of $X$ are scattered (e.g., if they are regular), and $\operatorname{pl}(X \backslash A) \neq \operatorname{pl}(X)$, we have locally closed $D \subset X$ with $D \backslash A$ scattered but $D$ not scattered. The dense-in-itself kernel $K$ is closed in $D . K \backslash A$ is scattered, so $K \backslash A$ is not dense in $K$. In the regular space $K$, this means there is a regular closed set $L \neq 0$ disjoint from $K \backslash A . L$ is dense in itself, contained in $A$, closed in $D$. So $L$ is locally closed in $X$ : there are closed sets $L_{\alpha}$ of $X$ contained in $L$ whose interiors relative to $L$ cover $L$. 
But any of those interiors that is nonempty is dense in itself, so its closure is dense in itself, closed in $X$, and contained in $A$.

In regular spaces, 2.7 gives the criterion for the general relationship:

2.8. For subspaces $Y, Z$ of a regular space $X, \operatorname{pl}(Y) \subset Z$ if and only if every subspace of $Y \backslash Z$ closed in $Y \cup Z$ is scattered.

Proof. Observe

$(\dagger)$ For a (sober) subspace $S$ of a space $T$ having a pointless part $\mathrm{pl}(T)$, $\operatorname{pl}(S)=S \wedge \operatorname{pl}(T)$.

Proof. Concerning "sober", pl( ) is defined in terms of sublocales, so only sober subspaces count. [If you prefer, define $\mathrm{pl}$ ( ) on spaces via sobrification.] Then $S \wedge \operatorname{pl}(T)$ is pointless and contained in $S$, so it is contained in $\operatorname{pl}(S)$. But $\operatorname{pl}(S)$ is contained in $S$ and in $\operatorname{pl}(T)$.

(††) In spaces having $\mathrm{pl}, \mathrm{pl}(Y \cup Z)=\mathrm{pl}(Y) \vee \mathrm{pl}(Z)$.

Proof. Note $Y \cup Z=Y \vee Z$, since it is the join of its points. So $\operatorname{pl}(Y \cup Z)=$ $\operatorname{pl}(Y \cup Z) \wedge(Y \vee Z)=[\operatorname{pl}(Y \cup Z) \wedge Y] \vee[\operatorname{pl}(Y \cup Z) \wedge Z]=\operatorname{pl}(Y) \vee \operatorname{pl}(Z)$.

But now $\operatorname{pl}(Y) \subset Z$ iff $\operatorname{pl}(Y \cup Z)=\operatorname{pl}(Z)$, and 2.5 applies (with $A=Y \backslash Z$, $X=Y \cup Z)$.

Remark. Paralleling $(\dagger \dagger), \operatorname{pl}(Y \wedge Z)=\operatorname{pl}(Y) \wedge \operatorname{pl}(Z)$-trivially. But $Y \wedge Z \neq$ $Y \cap Z$ in general.

2.9. $\mathbf{R}$ has $2^{\mathfrak{c}}$ nonisomorphic sublocales $\mathrm{pl}(X)(X \subset \mathbf{R})$.

Proof. By $2.7, \operatorname{pl}(\mathbf{R}) \approx \operatorname{pl}((0,1)) \approx \operatorname{pl}([0,1])$. There is a map of the ternary Cantor set $\mathbf{C}$ onto $[0,1]$ whose restriction off the endpoints of intervals of the complement is a homeomorphism to $[0,1]$ minus the dyadic rationals. The exceptional sets are countable, so totally imperfect; so $\operatorname{pl}(\mathbf{R}) \approx \operatorname{pl}(\mathbf{C})$. This is $\mathrm{pl}(\mathbf{C} \times \mathbf{C})$ by homeomorphism. The subspaces of $\mathbf{C} \times \mathbf{C}$ of the form $\mathbf{C} \times S$, $S \subset \mathrm{C}$, are $2^{\mathrm{c}}$ in number and by 2.8 , they all have different pointless parts in $\mathbf{C} \times \mathbf{C}$. Since a separable metrizable locale has at most $\mathbf{c}$ embeddings in $\mathbf{C} \times \mathbf{C}$ (at most c morphisms; for the frames are countably generated and of power c), the result follows.

There are partial results to the effect that $\operatorname{pl}(Y) \subset X$ is $\operatorname{pl}(Z \subset X)$; at least 2.10 here. It depends on the Lavrentiev theorem:

Any isomorphism between sublocales $A, B$ of completely metrizable locales $A^{-}, B^{-}$, extends to a homeomorphism between $G_{\delta}$ subspaces $C \supset A$ of $A^{-}$, $D \supset B$ of $B^{-}$.

This was proved by Hager $[\mathrm{H}]$ more generally (but he notes that if his spaces are paracompact, they must be completely metrizable).

2.10. Theorem. For spaces, $X$ completely metrizable and $E$ metrizable, each sublocale $P$ of $E$ isomorphic with $\mathrm{pl}(X)$ is $\mathrm{pl}(S)$ for a subspace $S$ of $E$ homeomorphic with a subspace of $X$ whose complement is totally imperfect. If $P=\operatorname{pl}(E), E \backslash S$ is also totally imperfect and $E \backslash S^{-}$is scattered. 
Proof. Let $F$ be a metric completion of $E$ and consider an isomorphism $i$ from $\operatorname{pl}(X)$ to a sublocale $P$ of $E \subset F$. There is a Lavrentiev extension $h: C \leftrightarrow D, C$ a $G_{\delta}$ of $X$ containing $\operatorname{pl}(X), D$ a $G_{\delta}$ of $F$ containing $P$. Since $\mathrm{pl}()$ takes the largest pointless part, $\operatorname{pl}(X)=\operatorname{pl}(C)$. Therefore the homeomorphism $h$ takes $\mathrm{pl}(X)$ to $\mathrm{pl}(D)=P$. But every subspace of $D \backslash E$ closed in $D$ is scattered, as it is completely metrizable and if not scattered it would contain a Cantor set $H$, closed in $F$; this would mean that the two open sets $F$ and $F \backslash H$ have the same trace on $E$ but not on $\operatorname{pl}(D) \subset E$, a contradiction. Then by $2.7, \operatorname{pl}(D \cap E)=\operatorname{pl}(D) . h^{-1}$ takes $D \cap E$ to a subspace of $X$ which contains $\mathrm{pl}(X)$, so its complement is totally imperfect. If $P$ is also $\operatorname{pl}(E), E \backslash D$ is similarly totally imperfect; and since $\operatorname{pl}(D \cap E)=P=\operatorname{pl}(E)$, $(D \cap E)^{-}$contains the dense-in-itself kernel of $E$.

2.11. Corollary. For separable completely metrizable spaces, $\operatorname{pl}(X) \approx \operatorname{pl}(Y)$ if and only if $X$ and $Y$ have homeomorphic subspaces with countable complements.

Proof. Sufficiency is evident. Conversely, if $\operatorname{pl}(X) \approx \mathrm{pl}(Y)$, this is the situation of 2.10 with $E$ also an absolute $G_{\delta}$. So $D \cap E$ is $G_{\delta}$, and the totally imperfect difference sets are relative $F_{\sigma}$ 's, absolute $G_{\delta \sigma}$ 's. Separable totally imperfect $G_{\delta}$ 's are countable, so $G_{\delta \sigma}$ 's are too.

2.12. Corollary. For $X$ completely metrizable and $E$ metrizable, if $\operatorname{pl}(X) \approx$ $\mathrm{pl}(E) \neq 0$ then $E$ is of the second category in itself.

Proof. The proof of 2.10 gives completely metrizable $D$ with $D \cap E$ dense in $E$ and $D \backslash E$ totally imperfect (and $D \cap E \neq 0$ ). Any countable union of nowhere dense sets in $D \cap E$ is contained in a countable union of closed nowhere dense sets in $D$, whose complement is then a dense $G_{\delta}$-thus not contained in $D \backslash E$. So $D \cap E$ itself is not such a union, but is second category. Since $E$ has a dense second category subspace, $E$ is second category.

On the other hand, $\mathrm{pl}(X)$ for $X$ complete can be isomorphic with $\mathrm{pl}(E)$ where $E$ is totally imperfect; in fact, it is (for every separable completely metrizable $X$, at least). For every separable metric space $X$ has at most c points. If fewer, $X$ is totally imperfect. If $\mathbf{c}$, then since $X$ contains at most c Cantor sets, each of power c, one can partition $X$ into two subsets $A, B$, each of which meets every Cantor set, so that each is totally imperfect; and by 2.7, if $X$ is complete, $\operatorname{pl}(A)=\operatorname{pl}(X)=\operatorname{pl}(B) \subset X$.

The countable complements in 2.11 are zero-dimensional, so they add 0 or 1 to the dimension of the subspace. But this generalizes, to perfectly normal spaces and a bit further to Dowker's totally normal spaces, or to any other class of normal spaces $X$ whose subspaces have covering dimension no greater than $\operatorname{dim} X$. [D]

2.13. For totally normal spaces $X, \operatorname{dim} \operatorname{pl}(X)$ is $\operatorname{dim} X$ or $\operatorname{dim} X-1$.

Proof. Since dim is monotone on subspaces it is monotone on sublocales, as follows. If $A$ is a sublocale of $X$, it is dense in $A^{-}$, which is a subspace. 
Any finite open covering $\left\{U_{i}\right\}$ of $A$ extends to $\left\{A^{-} \backslash\left(A \backslash U_{i}\right)^{-}\right\}$, a finite open (in $A^{-}$) covering of whatever it covers: a relatively open set $A^{*}$ between $A$ and $A^{-}$. So there is a finite open refinement covering $A^{*}$ of order at most $1+\operatorname{dim} A^{*} \leq 1+\operatorname{dim} X$, and its trace on $A$ refines $\left\{U_{i}\right\}$.

For the converse, observe that $\mathrm{pl}(X)^{-}$is the complement of a scattered open set. Hence $\operatorname{dim} X=\operatorname{dim} \operatorname{pl}(X)^{-}$(unless $\operatorname{pl}(X)$ is empty, which makes $\operatorname{dim} X$ zero). Put $X^{\prime}=\operatorname{pl}(X)^{-}$. Now for any finite open covering $\left\{V_{j}\right\}$ of $X^{\prime}$, by normality there is a shrinking $\left\{W_{j}\right\}$ with $W_{j}^{-} \subset V_{j}$. The trace of $\left\{W_{j}\right\}$ on $\operatorname{pl}(X)$ has a finite open refinement $\left\{U_{k}\right\}$ of order at most $1+\operatorname{dim} \operatorname{pl}(X)$. As before, $\left\{X^{\prime} \backslash\left(\operatorname{pl}(X) \backslash U_{k}\right)^{-}\right\}$is a finite open covering $\mathscr{C}$ of an open set $P$ between $\operatorname{pl}(X)$ and $X^{\prime}$. Since $\operatorname{pl}(X)$ is dense in $X^{\prime}$, any subset of $\mathscr{C}$ having a nonempty intersection has a nonempty intersection in $\mathrm{pl}(X)$, i.e. those $U_{k}$ 's meet; so we have not increased the order. $\mathscr{C}$ refines the trace of $\left\{V_{j}\right\}$ on $P$. $X^{\prime} \backslash P$ is scattered, so the trace of $\left\{V_{j}\right\}$ on it can be refined to a partition into relatively open-closed sets $Z_{m}$. Since $X^{\prime} \backslash P$ is closed, the $Z_{m}$ are closed and can be extended to disjoint open sets still each contained in one $V_{j}$. This gives an open refinement $\left\{V_{j}\right\}$ of order at most $2+\operatorname{dim} \operatorname{pl}(X)$, completing the proof.

We speak, above, of the dimension of the ghostly space $\mathrm{pl}(X)$, but not of its category: only, as in 2.12 , of the category of another space $E$ with $\operatorname{pl}(E) \approx$ $\mathrm{pl}(X)$. Why? It seems clear how second category locales $A$ should be defined: in the complete lattice of sublocales, $A$ is not a countable join of nowhere dense sublocales. This is easily seen to agree with Baire category for sober $T_{1}$ spaces. A non- $T_{1}$ space with a dense point is always a second category space but sometimes a first category locale, since nonsober spaces are not sublocales. This imperfection is not a serious drawback (how much Baire category theory is there outside $T_{1}$ spaces?), and I shall use this definition of category. However, for all I know, every nonzero $\mathrm{pl}(X)$ may be second category. At least:

\subsection{If $X$ is metrizable and $\operatorname{pl}(X) \neq 0$ then $\operatorname{pl}(X)$ is second category.}

Proof. Since $\operatorname{pl}(X)$ is contained in the dense-in-itself kernel $D$ and is $\operatorname{pl}(D)$, we may assume $X$ is dense in itself. Given countably many nowhere dense parts $K_{i}(i \geq 1)$ of $\mathrm{pl}(X)$, we have closed nowhere dense subspaces $L_{i}=K_{i}^{-}$ of $X$. We now prove

( $\ddagger$ For any sequence of closed nowhere dense sets $L_{i}$ in a dense-in-itself metric space $X$ and any dense subset $P$ of $X$, there is a closed dense-in-itself subset $H$ of $X$ such that each $H \cap L_{i}$ is finite and $H \cap P$ is dense in $H$.

The dense set $P$ has nothing to do with 2.14; we want it for 2.18.b.

Proof of $(\ddagger)$. Choose $x_{0} \in P$. Choose a surjective $\infty$-to-1 function $f: \omega \backslash\{0\} \rightarrow$ $\omega$ satisfying $f(n)<n$. We shall successivt'y choose $x_{n}$ within a small distance $\varepsilon_{n}$ of $x_{f(n)}$, in (dense) $P$ but not in (nowhere dense) $L_{1} \cup \cdots \cup L_{n}$. In fact we require $(1) \varepsilon_{n} \leq d\left(x_{n-1}, x_{f(n-1)}\right) / 3 . \quad\left(\varepsilon_{1}=1\right.$, say. $)$ Also, for each 
$i \leq f(n),(2) \varepsilon_{n} \leq d\left(x_{f(n)}, L_{i}\right) / 3$. (That is $>0$, as $x_{f(n)}$ was chosen outside these $L$ 's.) Finally $S$ is the set of $x_{i}, H=S^{-}$. Clearly $H$ is closed dense-in-itself, $H \cap P \supset S$ is dense in it, and each $S \cap L_{i}$ is finite.

I claim $S^{-} \cap L_{i}=S \cap L_{i}$. Consider the natural numbers partially ordered by $f$ : the ancestors of $n$ are $f(n), f^{2}(n), \ldots, 0$, and it is their descendant. Consider any convergent sequence of distinct elements $x_{n_{k}}$. Infinitely many $n_{k}$, in fact all of them, are descendants of 0 . Either there is a genetic sequence $0 \rightarrow m_{1} \rightarrow m_{2} \rightarrow \cdots$ of natural numbers all of which have infinitely many descendants $n_{k}$, or there is a maximal such $m$. In the latter case, the next generation $f^{-1}(m)$ has infinitely many members $r_{i}$ such that $r_{i}$ or one of its descendants is an $n_{k}$. But observe, first, $d\left(x_{r_{i}}, x_{m}\right) \rightarrow 0$; and because of (1), any descendants $y_{i}$ of $x_{r_{i}}$ also converge to $x_{m}$. For the other case, first, for each $i$, almost all $m_{j}$ are $\geq i$, so $x_{m_{j}}$ is not in $L_{i}$; and because of (2), when $m>i$, all descendants of $x_{m}$ are at least half as far from $L_{i}$. Thus in neither case is there a limit in $L_{i} \backslash S$. So ( $\left.\ddagger\right)$ holds, and 2.14 holds since $\bigvee K_{i} \leq \mathrm{pl}(X-H)$, which is not $\mathrm{pl}(X)$ since $\mathrm{pl}(H) \neq 0$.

The result 2.16 below seems feeble compared with 2.14 ; but metrizability is hard to do without, and 2.16 seems worth mentioning. First:

2.15. A space $X$ which is a $G_{\delta}$ in a compact Hausdorff space and is not scattered has a compact dense-in-itself subspace.

Proof. Let $X \subset Y$ be the countable intersection of open sets $U_{i}$ in compact Hausdorff $Y$. Let $A(0)$ and $A(1)$ be disjoint regular closed sets contained in $U_{1}$, whose interiors meet the dense-in-itself part of $X$. Inductively, having $2^{n}$ disjoint regular closed sets $A(\sigma)$, indexed by all sequences $\sigma$ of $n$ terms 0 or 1 , whose interiors meet the dense-in-itself part of $X$, take two such sets $A(\sigma, 0)$ and $A(\sigma, 1)$ interior to $A(\sigma)$ and contained in $U_{n+1}$ (for each $\sigma$ ); this is always possible and yields nested sets $A(\sigma)$ for all finite $0-1$ sequences $\sigma$. For every point $\pi \in 2^{\omega}$, the intersection $A(\pi)$ of $A(\sigma)$ over all initial segments $\sigma$ of $\pi$ is nonempty by compactness and contained in the $G_{\delta}$ set $X$. The union $A$ of all $A(\pi)$ is compact (as an intersection of compact unions $A(0) \cup A(1)$ etc.). The map $f: A \rightarrow 2^{\omega}$ taking each $A(\pi)$ to $\pi$ is continuous by construction. Therefore $[R] A$ is not scattered, and its dense-in-itself part proves the assertion.

2.16. If $X$ is a dense-in-itself $G_{\delta}$ in a compact Hausdorff space, $\mathrm{pl}(X)$ is second category.

Proof. $X$ is a dense $G_{\delta}$ in a compact dense-in-itself space $Y$ (take its closure). So $Y \backslash X$ is a countable union of nowhere dense closed sets. Suppose $\operatorname{pl}(X)$ is the join of nowhere dense $A_{i} \subset \mathrm{pl}(X)$; then $(Y \backslash X) \cup\left(\bigcup A_{i}^{-}\right)$is an $F_{\sigma}$ subspace $S$ of $Y$ containing $Y \backslash X$ and $\operatorname{pl}(X)$. Moreover, the subspace intersection $I$ of $S$ and $X$ contains $\mathrm{pl}(X)$, since each $A_{i} \subset X \cap A_{i}^{-}$, which is closed in $X$ and therefore a subspace. Then by 2.7 , every subspace of $X \backslash I$ closed in $X$ 
is scattered. But $X \backslash I=Y \backslash S$, a $G_{\delta}$, so by 2.15 it is scattered. In particular, $Y \backslash S$ is empty or has an isolated point. $Y \backslash S$ empty would mean $Y$ is first category; an isolated point $p$ of $Y \backslash S$ would have an open neighborhood $U$ containing no other point of $Y \backslash S$, so the locally compact dense-in-itself space $U \backslash\{p\}$ would be first category; both impossible.

Now, all I know about $\operatorname{pl}(Q)$, the pointless part of the rationals. First,

2.17.a. If $X$ is metrizable and $\operatorname{pl}(X) \approx \operatorname{pl}(Q)$ then $X$ is totally imperfect.

Proof. If $X$ is not totally imperfect it contains a Cantor set $\mathbf{C}, \operatorname{pl}(\mathbf{C}) \subset \operatorname{pl}(X)$ is isomorphic with a sublocale $P$ of $\operatorname{pl}(Q)$, and by $2.10, P$ is $\operatorname{pl}(T)$ for some subspace $T$ of $Q$. But $\operatorname{pl}(T)$ is 0 or isomorphic with $\operatorname{pl}(Q)$, unlike $\operatorname{pl}(\mathbf{C})$ (2.12).

Second:

2.17.b. A (complete) metric space $X$ can have a sublocale $P \approx \mathrm{pl}(Q)$ which is not $\mathrm{pl}(S)$ for any subspace $S$ of $X$.

Proof. As noted in $\left[I_{1}\right]$ (and easily seen from 2.4), in the real line $\mathbf{R}$ the space of irrationals $\mathbf{R} \backslash Q$ contains the sublocale $\mathrm{pl}(Q)$. But $\mathbf{R}$ has no subspace $S$ disjoint from $Q$ with $\mathrm{pl}(S)=\mathrm{pl}(Q)$. For, if $S \supset \mathrm{pl}(Q)$ then $S$ is dense in $\mathbf{R}$. But we can list the elements $x_{i}$ of $Q$ and enclose them in open intervals $I_{i}$ of length $\varepsilon_{i}$, whose union will be an open set $U$ of measure $<1$; let us see what else the construction requires. $\mathbf{R} \backslash U$ will be closed and $(\mathbf{R} \backslash U) \cap Q$ scattered, in fact empty; if $(\mathbf{R} \backslash U) \cap S$ is dense in itself, this will show $Q$ does not contain $\operatorname{pl}(S)$. For this, clearly, no two $I_{i}$ can abut, leaving an isolated point of $S$ between them. Further, we list the elements $V_{j}$ of a basis of open intervals of $\mathbf{R}$ and, in attending alternately to $x_{1}, V_{1}, x_{2}, V_{2}, \ldots$, when attending to $V_{j}$, if it is not already covered by $U$, mark two points of $S \cap V_{j}$ to be left out of $U$. The finite union of nonabutting intervals already put in $U$ has regular closed complement, so the construction is possible.

Recall (from several pages ago) the first pullback lemma, 1.9. It is about open/closed/locally closed $/ O_{\delta}$ sublocales. Nothing like it holds for $F_{\sigma}$ 's. $Q$ is $F_{\sigma}$ in $\mathbf{R}$ (or anywhere); but $\operatorname{pl}(Q)$ is not only not a countable join of closed parts of $\mathrm{pl}(\mathbf{R})$, it is not a join of them at all. Nor of $O_{\delta}$ parts; moreover,

2.18.a. If $Q^{\prime}$ has $\mathrm{pl}$ and $\mathrm{pl}(\mathbf{R})$ cannot be embedded in $\mathrm{pl}\left(Q^{\prime}\right)$, then for any absolute $G_{\delta}$ space $X$, a sublocale of $\mathrm{pl}(X)$ isomorphic with $\mathrm{pl}\left(Q^{\prime}\right)$ contains no nonzero $O_{\delta}$.

For the $O_{\delta}$ 's of $\mathrm{pl}(X)$ are $\mathrm{pl}(S), S$ a $G_{\delta}$ in $X$; if $\mathrm{pl}(S)$ is nonzero, $S$ contains $\mathrm{C}$ and $\operatorname{pl}(S)$ contains $\mathrm{pl}(\mathbf{C}) \approx \operatorname{pl}(\mathbf{R})$.

Turning from non- $O_{\delta \sigma}$ 's to non- $F_{\sigma \delta}$ 's, we have

2.18.b. If $T$ is a dense subspace of a completely metrizable space $X$, then $\mathrm{pl}(T) \subset \mathrm{pl}(X)$ either contains a dense $O_{\delta}$ or is not $F_{\sigma \delta}$. 
Proof. The core is

( $\ddagger)$ A dense embedding of metrizable $T$ in completely metrizable $X$ induces an embedding of $\mathrm{pl}(T)$ in $\mathrm{pl}(X)$ such that every $F_{\sigma}$ sublocale containing $\mathrm{pl}(T)$ contains a dense open sublocale.

Proof. We may assume $X$ is dense in itself. For any $F_{\sigma}$ sublocale $\bigvee \operatorname{pl}\left(J_{i}\right)$ of $\operatorname{pl}(X)$ that contains $\operatorname{pl}(T), X \backslash \cup J_{i}$ is nowhere dense; for if it were dense in nonempty open $U$, the closed sets $L_{i}=J_{i} \cap U^{-}$would be nowhere dense and subject to $(\ddagger): U^{-}$would have a closed dense-in-itself subset $H=(H \cap T)^{-}$ with all $H \cap L_{i}$ finite. This means $\operatorname{pl}(X \backslash H)$ contains all $\operatorname{pl}\left(J_{i}\right)$ but not $\operatorname{pl}(T)$, a contradiction. So the closure of $X \backslash \bigcup J_{i}$ has void interior, i.e. $W=\left(\bigcup J_{i}\right)^{\circ}$ is dense. Next we show that a symmetric difference $U \Delta V$ of two open sets of $X$ which meets $\operatorname{pl}(W)$ meets some $\operatorname{pl}\left(J_{i}\right)$. For if the absolute $G_{\delta}$ set $U \Delta V$ has nonscattered intersection with absolute $G_{\delta} W$, the intersection contains a Cantor set $\mathbf{C}$, which is not a countable union of scattered (hence nowhere dense) subsets $\mathbf{C} \cap J_{i}$; some $\mathbf{C} \cap J_{i}$ has nonzero $\operatorname{pl}\left(\mathbf{C} \cap J_{i}\right) \subset(U \Delta V) \cap \operatorname{pl}\left(J_{i}\right)$. But this means $\bigvee \mathrm{pl}\left(J_{i}\right)$ contains $\mathrm{pl}(W)$, as claimed.

2.18.b is immediate; $F_{\sigma}$ 's containing $\mathrm{pl}(T)$ contain dense opens, so $F_{\sigma \delta}$ 's containing it contain dense $O_{\delta}$ 's $\mathrm{pl}\left(\cap W_{i}\right)$.

Remark. ( $\ddagger)$ can be restated: in these $\mathrm{pl}(X)$, an $F_{\sigma}$ containing a dense $\mathrm{pl}(T)$ has dense interior. These $\mathrm{pl}(X)$ (to anticipate 2.19) are the pointless metrizable absolute $O_{\delta}$ 's. $\operatorname{pl}(T)$ is essential; the $F_{\sigma}$ cannot be merely dense. (Proof, imitate a countable dense set in $X$ using a little Cantor set for each point.) But I do not know if $\mathrm{pl}(T)$ ends up in the interior.

2.18.a, or 2.18.b, shows that the first sentence of 1.9 fails for $F_{\sigma}$ 's. As for the second:

2.18.c. There exists an $F_{\sigma}$ sublocale of $\mathrm{pl}(Q)$ which is not $\mathrm{pl}(S)$ for any subspace $\left(=F_{\sigma}\right.$ sublocale) $S$ of $Q$.

Proof. Observe, $Q$ is homeomorphic with $Q \times Q$. That's a union of $\omega$ vertical sections, which are closed. The pointless parts of the vertical sections are closed in $\operatorname{pl}(Q \times Q)$-which is just $\operatorname{pl}(Q)$; take their union, an $F_{\sigma}$ in $\operatorname{pl}(Q)$. If it were the trace of an $F_{\sigma}$ in $Q(=Q \times Q)$, it would be second category in itself by 2.14 , which it plainly is not.

A further detail:

2.18.d. There is an $F_{\sigma}$ in $\mathrm{pl}(\mathbf{R})$ which is not $\mathrm{pl}(S)$ for any subspace $S\left(F_{\sigma}\right.$ or not) of $\mathbf{R}$.

For there is an ascending sequence of closed parts whose join is first category in itself.

2.19. Theorem. For a completely metrizable space $X, \mathrm{pl}(X)$ is an $O_{\delta}$ sublocale of every pointless metrizable locale containing it. A pointless metrizable 
locale which is an $O_{\delta}$ in every pointless metrizable extension is $\mathrm{pl}(X)$ for some completely metrizable space $X$.

Proof. If $\mathrm{pl}(X)$ is isomorphic with $P \subset E, E$ pointless metrizable, let $F$ be a metric completion of $E$. There is a Lavrentiev extension of $i: \operatorname{pl}(X) \approx P$ to a homeomorphism $h$ of containing $G_{\delta}$ 's $C$ in $X, D$ in $F . P=\operatorname{pl}(X)=\operatorname{pl}(C)$, so $h$ takes $P$ isomorphically to $\operatorname{pl}(D) . \operatorname{pl}(D)$ is $O_{\delta}$ in $\operatorname{pl}(F)$, hence also in $E \subset \operatorname{pl}(F)$. Now suppose merely that $P$ is an $O_{\delta}$ in $\mathrm{pl}(F)$ for a metric completion $F$; then by $1.9, P=\operatorname{pl}(D)$ for some $O_{\delta}$ sublocale $D$ of $F$, but $D$ is spatial and completely metrizable.

2.20. For every $G_{\delta}$ part $X$ of $\mathbf{R}, \operatorname{pl}(X)$ is 0 or isomorphic with $\mathrm{pl}(\mathbf{R})$.

Proof. Since the dense-in-itself kernel $K$ of $X$ is a $G_{\delta}$, it suffices to show that every dense-in-itself $G_{\delta}$ part $K$ has $\operatorname{pl}(K) \approx \mathrm{pl}(\mathbf{R})$, or equivalently, $\approx \operatorname{pl}(\mathbf{R} \backslash Q)$. But $K$ minus a countable dense subset $S$ of $K$ is homeomorphic with $\mathbf{R} \backslash Q$. This will suffice, since $\operatorname{pl}(K \backslash S)=\operatorname{pl}(K)$ by 2.7. It is true by the Alexandroff-Urysohn characterization [AU] of $\mathbf{R} \backslash Q$ : a separable metric 0-dimensional absolute $G_{\delta}$ (obvious for $K \backslash S$ ), which is nowhere locally compact. $K \backslash S$ is nowhere locally compact because $S$ is dense in $K$.

2.21. $\mathrm{pl}(\mathbf{R})$ is characterized, up to isomorphism, as a nonzero pointless zerodimensional separable metrizable locale which is $O_{\delta}$ in every pointless metrizable extension.

Proof. Given such a locale $F$, it has a zero-dimensional metrizable compactification $\left[\mathrm{I}_{3}\right]$, so it is embeddable in $\mathbf{R}$. Being pointless, it goes into $\mathrm{pl}(\mathbf{R})$, where it is $O_{\delta}$; so it is $\mathrm{pl}(X)$ for some $G_{\delta}$ subspace $X$ of $\mathbf{R}$, and by 2.20 it is $\approx \mathrm{pl}(\mathbf{R})$.

Among spaces, a metrizable absolute $G_{\delta}$ is a $G_{\delta}$ in any completely regular space in which it is dense. Not among pointless locales.

(§) Every nonzero pointless metrizable locale is dense but not $O_{\delta}$ in some completely regular pointless locale.

Proof. We may assume the given locale $A$ is $\mathrm{pl}(M)$ for some dense-in-itself completely metrizable space $M$. For each finite subset $F$ of $M$, let $M_{F}$ be the subset of $\beta(M \backslash F)$ which maps to $M$ under the natural map $\beta(M \backslash F) \rightarrow \beta M$. Let $L$ be the inverse limit of the $M_{F}$ (mapping $M_{G} \rightarrow M_{F}$, for $G \supset F$, by restriction of $\beta$ of $M \backslash G \subset M \backslash F)$; let $B=\operatorname{pl}(\beta L) . A \subset M$ is contained in each $M \backslash F$, so naturally embedded in $M_{F}$ and in $L \subset \beta L$. Since $A$ is pointless it is contained in $B$. If it were $O_{\delta}$ there, it would be the trace on $B$ of a $G_{\delta}$ set $D$ of $L$. Then $\beta L \backslash D$ would be a countable union of closed sets $H_{i}$, all disjoint from $A$. But if closed $H \subset \beta L$ is disjoint from $A$, the natural map $f: \beta L \rightarrow \beta M$ cannot take $H$ to a set containing a Cantor set $C \subset M$. For $K=H \cap f^{-1}(C)$ would be compact with $C$ as a quotient, so nonscattered [R]; and in the inverse mapping system we have $\operatorname{pl}(C) \subset \mathrm{pl}(M)=A$ mapping 
isomorphically throughout, and $\mathrm{pl}(K)$ projecting into it at every stage, so into $\mathrm{pl}(C)$ in the limit, i.e. $0 \neq \mathrm{pl}(K) \subset A$, a contradiction. We conclude that each $f\left(H_{i}\right) \cap M$ is scattered. But then they are nowhere dense; the union misses $f^{-1}(p)$ for some point $p$ of $M, D$ contains $f^{-1}(p)$, and $D \wedge B=\operatorname{pl}(D) \supset$ pl $\left.f^{-1}(p)\right)$, so $D \wedge B \neq A$.

2.22. There are c nonisomorphic connected dense $O_{\delta}$ sublocales $\mathrm{pl}\left(X_{\alpha}\right)$ of $\mathrm{pl}\left(\mathbf{R}^{2}\right)$.

Proof. The indices $\alpha$ will be the subsets of $\omega_{0} \backslash\{0\}$. We first describe nonhomeomorphic compact sets $B_{\alpha} . B_{0}$ (0 for the null set) is $\left(\omega^{\omega}+1\right) \times I$, where $\omega=\omega_{0}, \omega^{\omega}+1$ has the order topology, and $I$ is a closed interval. $\omega^{\omega}+1$ can be embedded in $\mathbf{R}$, and $B_{0}$ can be embedded in $\mathbf{R}^{2}$. For a general set $\alpha$ of positive integers, modify $B_{0}$ to $B_{\alpha}$ by lengthening its $\omega^{n}$ th interval $\left\{\omega^{n}\right\} \times I$ for each $n \in \alpha$. (So the limit of $\{\beta\} \times I$ as $\beta \rightarrow \omega^{n}$ is a proper subinterval. For compactness, either do not lengthen them very much or lengthen $\left\{\omega^{\omega}\right\} \times I$ too.) Evidently the $B_{\alpha}$ are topologically distinct and embeddable in $\mathbf{R}^{2}$.

Let $V_{\alpha}$ be a countable discrete set in $\mathbf{R}^{2} \backslash B_{\alpha}$ whose derived set is $B_{\alpha}$; but that is just to mark where to put $W_{\alpha}$, which is a topological sum of $\omega$ Cantor sets in $\mathbf{R}^{2} \backslash B_{\alpha}$ with $W_{\alpha}^{-} \backslash W_{\alpha}=B_{\alpha}$. This makes $X_{\alpha}=\mathbf{R}^{2} \backslash W_{\alpha}$ a dense $G_{\delta}$, so $\operatorname{pl}\left(X_{\alpha}\right)$ is dense $G_{\delta}$ in $\mathrm{pl}\left(\mathbf{R}^{2}\right)$. If we also choose $W_{\alpha}$ to approach $B_{\alpha}$ (locally) only from one side, then $X_{\alpha}$ will be a union of closed 2-cells and $\operatorname{pl}\left(X_{\alpha}\right)$ connected. ( $\operatorname{pl}\left(X_{\alpha}\right)$ would be connected without this precaution, but it is harder to see.)

By $2.11, \operatorname{pl}\left(X_{\alpha}\right) \approx \operatorname{pl}\left(X_{\beta}\right)$ iff they have homeomorphic subspaces $S_{\alpha}, S_{\beta}$ with countable complements. Now $S_{\alpha}$ and $S_{\beta}$ are each $S^{2}$ minus a zerodimensional subspace. So their Freudenthal compactifications are $S^{2}$; and $S_{\gamma}$ $(\gamma=\alpha, \beta)$ determines the remainder $R_{\gamma}=S^{2} \backslash S_{\gamma}$ as a subspace of $S^{2}$. But the set of accumulation points of $R_{\gamma}$ (points at which $R_{\gamma}$ is not locally countable) is $W_{\gamma}^{-}$, the nondegenerate components constitute $B_{\gamma}$, so $S_{\alpha}$ and $S_{\beta}$ are not homeomorphic.

2.23. For each connected dense open subset $X$ of $\mathbf{R}^{2}, \operatorname{pl}(X)$ is isomorphic with $\mathrm{pl}\left(\mathbf{R}^{2}\right)$ or with $\mathrm{pl}\left(\mathbf{R}^{2} \backslash \mathbf{C}\right)$.

Proof sketch. The Freudenthal compactification of $X$, or of $X$ minus a countable set, is $S^{2}$. For clearly the countable set does not affect it, and the point is that $S^{2} \backslash X$ (closed nowhere dense nonseparating) is the intersection of a shrinking sequence of nice neighborhoods: all $X$ knows is how many components $S^{2} \backslash X$ has. With the floating countable set, all $\mathrm{pl}(X)$ knows is whether $S^{2} \backslash X$ has uncountably many components.

There is nothing like 2.23 in $\mathbf{R}^{3}$; one can remove a sequence of different knots in $\mathbf{c}$ ways. 
2.24. No nonzero pointless metrizable locale is an $F_{\sigma}$ in every pointless metrizable extension.

Proof. Consider $A \neq 0$ embedded in a completely metrizable space $X$. If $A$ is $F_{\sigma}$ in $\mathrm{pl}(X)$, it contains $\mathrm{pl}(H)$ for some nonscattered $H$ closed in $X$; hence $A$ has a closed sublocale $B=\operatorname{pl}(\mathbf{C}), \mathbf{C} \subset X$. Now $\operatorname{pl}(\mathbf{C})=\operatorname{pl}(\mathbf{C} \backslash Q)$, and $\mathrm{C} \backslash Q$ is homeomorphic with its square $S \subset \mathrm{C}^{2}$. Let $Y$ be the metrizable space $X \backslash Q$-the copy of $Q$ in $\mathrm{C}$ that we are using-and let $Z$ be the union of $Y$ and $\mathbf{C}^{2}$ with the two copies of $\mathbf{C} \backslash Q$ identified. This is a metrizable space; it is easy to construct it via an embedding of $\mathbf{C}^{2}$ in an absolute retract and

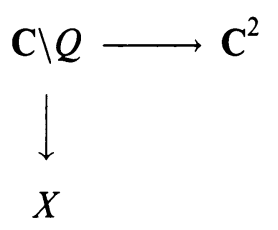

The closure of $B$ in $Z$ is $\mathbf{C}^{2}$. B is not an $F_{\sigma}$ in $\mathrm{pl}\left(\mathbf{C}^{2}\right)$; for $B$ is second category, and a closed set of $\mathbf{C}^{2}$ which is not nowhere dense has a perfect subset disjoint from the dense subset $S$. Hence $A$ is not $F_{\sigma}$ in $\operatorname{pl}(Z)$.

\section{REFERENCES}

[AU] P. S. Alexandroff and P. S. Urysohn, Nulldimensionale Punktmengen, Math. Ann. 98 (1927), 89-106.

[D] C. H. Dowker, Local dimension of normal spaces, Quart. J. Math. (Oxford) 6 (1955), 101120.

[H] A. W. Hager, Isomorphisms of some completions of $C(X)$, Topology Proc. 4 (1979), 407435 (1980).

[I $I_{1} \quad$ J. Isbell, Atomless parts of spaces, Math. Scand. 31 (1972), 5-32.

$\left[\mathrm{I}_{2}\right] \ldots$ - Function spaces and adjoints, Math. Scand. 36 (1975), 317-339.

$\left[\mathrm{I}_{3}\right] \ldots$, Graduation and dimension in locales, Aspects of Topology, London Math. Soc. Lecture Notes 93, 1985, pp. 195-210.

$\left[\mathrm{I}_{4}\right] \quad \ldots$, d-final continua, Proc. Amer. Math. Soc. 104 (1988), 953-964.

$\left[\mathrm{J}_{1}\right] \quad$ P. T. Johnstone, Stone spaces, Cambridge Univ. Press, 1982.

$\left[\mathrm{J}_{2}\right] \quad$ - Fibrewise separation axioms for locales, preprint.

[K] K. Kuratowski, Topologie, vol. I, 4th ed., Warsaw, 1958.

[M] D. Montgomery, Non-separable metric spaces, Fund. Math. 25 (1935), 527-534.

[NR] S. B. Niefield and K. I. Rosenthal, Spatial sublocales and essential primes, Topology Appl. 26 (1987), 263-269.

[R] W. Rudin, Continuous functions on compact spaces without perfect subsets, Proc. Amer. Math. Soc. 8 (1957), 39-42.

Department of Mathematics, State University of New York at Buffalo, Buffalo, NEW YORK 14214 